This item was submitted to Loughborough's Research Repository by the author.

Items in Figshare are protected by copyright, with all rights reserved, unless otherwise indicated.

\title{
Universal exciton size in organic polymers is determined by nonlocal orbital exchange in time-dependent density functional theory
}

\section{PLEASE CITE THE PUBLISHED VERSION}

https://doi.org/10.1021/acs.jpclett.7b00157

\section{PUBLISHER}

(C) American Chemical Society (ACS)

\section{VERSION}

AM (Accepted Manuscript)

\section{PUBLISHER STATEMENT}

This work is made available according to the conditions of the Creative Commons Attribution-NonCommercialNoDerivatives 4.0 International (CC BY-NC-ND 4.0) licence. Full details of this licence are available at: https://creativecommons.org/licenses/by-nc-nd/4.0/

\section{LICENCE}

CC BY-NC-ND 4.0

\section{REPOSITORY RECORD}

Mewes, Stefanie A., Felix Plasser, and Andreas Dreuw. 2019. "Universal Exciton Size in Organic Polymers Is Determined by Nonlocal Orbital Exchange in Time-dependent Density Functional Theory". figshare. https://hdl.handle.net/2134/32236. 


\title{
Universal Exciton Size in Organic Polymers is Determined by Non-local Orbital Exchange in TDDFT
}

\author{
Stefanie A. Mewes, ${ }^{*, \dagger}$ Felix Plasser,,$\ddagger$ and Andreas Dreuw ${ }^{*, \dagger}$ \\ $\dagger$ Interdisciplinary Center for Scientific Computing, Ruprecht-Karls University, Im \\ Neuenheimer Feld 205A, D-69120 Heidelberg, GERMANY \\ $\ddagger$ Institute for Theoretical Chemistry, Faculty of Chemistry, University of Vienna, \\ Währingerstr. 17, A-1090 Wien, AUSTRIA
}

E-mail: stefanie.mewes@iwr.uni-heidelberg.de; dreuw@uni-heidelberg.de 


\begin{abstract}
The exciton size of the lowest singlet excited state in a diverse set of organic $\pi$ conjugated polymers is studied and found to be a universal, system-independent quantity of approximately $7 \AA$ in the single chain picture. With time-dependent density functional theory (TDDFT), its value as well as the overall description of the exciton is almost exclusively governed by the amount of non-local orbital exchange. This is traced back to the lack of the Coulomb attraction between the electron and hole quasiparticles in pure TDDFT, which is reintroduced only with the admixture of non-local orbital exchange.
\end{abstract}

\title{
Graphical TOC Entry
}

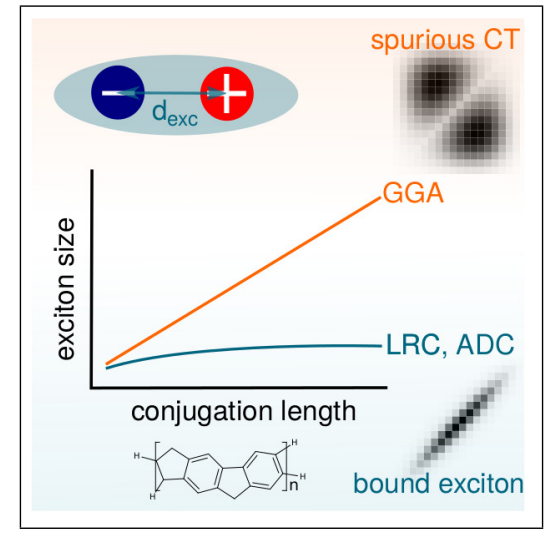


Quantum-chemical methods applied on modern computer architectures allow to investigate excited states of ever larger molecules. ${ }^{1-3}$ Despite the rapid developments on the computational side, the tools to analyze excited-state properties of these large systems are in practice often limited to a visual inspection of frontier orbitals. As an example, for a $\pi \rightarrow \pi^{*}$ excitation it seems at first sufficient to analyze the highest occupied (HOMO) and the lowest unoccupied molecular orbitals (LUMO) as they usually represent large parts of the excitation process. In extended $\pi$-systems, however, electron-hole correlation effects gain substantial importance and the assumption underlying MO theory that the excited electron is decoupled from the excitation hole is no longer true. ${ }^{4}$ Moreover, it becomes crucial to adopt a picture of coupled electron-hole pairs i.e. excitons, a concept which is central to the description of excited states in solid-state physics. ${ }^{5,6}$

In the following, evidence is provided for the necessity to move to a correlated electronhole description to investigate excitons in the context of quantum-chemical calculations of large molecular systems. ${ }^{4,7,8}$ Specifically, the onset of excitonic properties is studied for $\pi$ conjugated systems of varying size to illustrate how excitonic effects emerge. This aspect is of high importance in the discussion of localization effects in finite $\pi$-systems, a topic that is intensely debated. ${ }^{9-13}$ While previous work was focused on a qualitative rationalization of excitonic effects, ${ }^{14-17}$ a quantitative perspective is adopted here which allows to decompose excitonic effects in large conjugated $\pi$-systems on different levels. Furthermore, a hierarchy of exchange-correlation (xc) functionals is investigated in terms of their ability to describe excitonic properties, i.e. the electron-hole interaction, which turns out to crucially depend on the amount of non-local orbital exchange.

In linear-response time-dependent density functional theory (TDDFT), ${ }^{1,3}$ Casida's pseudoeigenvalue equation is solved

$$
\left(\begin{array}{cc}
\mathbf{A} & \mathbf{B} \\
\mathbf{B}^{*} & \mathbf{A}^{*}
\end{array}\right)\left(\begin{array}{l}
\mathbf{X} \\
\mathbf{Y}
\end{array}\right)=\omega\left(\begin{array}{rr}
\mathbf{1} & \mathbf{0} \\
\mathbf{0} & -\mathbf{1}
\end{array}\right)\left(\begin{array}{l}
\mathbf{X} \\
\mathbf{Y}
\end{array}\right)
$$


where $\omega$ is the excitation energy, and $\mathbf{X}$ and $\mathbf{Y}$ are the excitation and de-excitation amplitudes. The elements of the $\mathbf{A}$ matrix in the case of a hybrid functional are given as

$$
A_{i a, j b}=\delta_{i j} \delta_{a b}\left(\epsilon_{a}-\epsilon_{i}\right)+(i a \mid j b)-c_{\mathrm{HF}}(i j \mid a b)+\left(1-c_{\mathrm{HF}}\right)\left(i a\left|f_{x c}\right| j b\right)
$$

and the elements of the $\mathbf{B}$ matrix possess a similar structure with permuted indices. The indices $i, j$ and $a, b$ pertain to occupied and virtual orbitals, respectively. The $\epsilon_{i}$ and $\epsilon_{a}$ values denote the orbital energies, $c_{\mathrm{HF}}$ marks the fraction of non-local orbital exchange, and $f_{x c}$ is the $x c$-kernel of the employed functional. To connect this equation to the quasi-particle picture, ${ }^{18}$ we relabel the occupied orbitals involved as "hole" and the virtual orbitals as "electron". In this picture, the second term in Eq. (2) $(i a \mid j b)$, which is the response of (i.e. originates from) the Coulomb interaction in DFT takes the form of an exchange repulsion between the electron and hole. The third term $c_{\mathrm{HF}}(i j \mid a b)$, the response of the non-local exchange interaction takes the form of a Coulomb attraction. Hence, in TDDFT $c_{\mathrm{HF}}$ can be interpreted as charge screening parameter, c.f. Ref. 19. In the case of $c_{\mathrm{HF}}=1$ the electronhole pair experiences the full Coulomb attraction, while it is screened for lower values and disappears for $c_{\mathrm{HF}}=0$. The fourth term depends specifically on the functional and has no such simple interpretation. If range-separated functionals are used, Eq. (2) becomes somewhat more involved. In a nutshell, the more non-local orbital exchange is employed, the stronger becomes the electron-hole Coulomb attraction.

A new perspective on the results of Eq. (1) can be obtained employing exciton analysis. ${ }^{7,8,20,21}$ For this purpose, an effective exciton wavefunction is constructed using the excitation and de-excitation amplitudes

$$
\chi_{e x c}\left(r_{h}, r_{e}\right)=\sum_{i}^{o c c} \sum_{a}^{v i r t}\left[X_{i a} \phi_{i}\left(r_{h}\right) \phi_{a}\left(r_{e}\right)+Y_{i a} \phi_{a}\left(r_{h}\right) \phi_{i}\left(r_{e}\right)\right] .
$$

$\phi_{i}$ and $\phi_{a}$ refer to occupied and virtual molecular orbitals, respectively, and $r_{h}$ and $r_{e}$ are the coordinates of the electron and hole quasi-particles. ${ }^{4}$ To characterize excitons, the ex- 
pectation value of an operator $\hat{O}$ can be evaluated as

$$
\langle\hat{O}\rangle=\frac{\left\langle\chi_{e x c}|\hat{O}| \chi_{e x c}\right\rangle}{\left\langle\chi_{e x c} \mid \chi_{e x c}\right\rangle}
$$

To measure the spatial extent of the hole generated during excitation, the hole size is computed as

$$
\sigma_{h}=\sqrt{\left\langle r_{h}^{2}\right\rangle-\left\langle r_{h}\right\rangle^{2}}
$$

The exciton size depends on the joint electron-hole position, and is analyzed as the rootmean-square (rms) electron-hole distance

$$
d_{e x c}=\sqrt{\left\langle\left|r_{e}-r_{h}\right|^{2}\right\rangle} .
$$

Furthermore, linear electron-hole correlation effects are investigated employing a correlation coefficient similar to Pearson correlation as

$$
R_{e h}=\frac{\operatorname{COV}\left(r_{h}, r_{e}\right)}{\sigma_{h} \sigma_{e}}
$$

with the covariance defined as

$$
\operatorname{COV}\left(r_{h}, r_{e}\right)=\left\langle r_{h} \cdot r_{e}\right\rangle-\left\langle r_{h}\right\rangle \cdot\left\langle r_{e}\right\rangle
$$

$R_{e h}$ ranges from -1 to +1 , where negative values correspond to anti-correlation, i.e. a dynamical avoidance of electron and hole in space, 0 indicates no linear electron-hole correlation (which is the standard assumption in the MO-based picture), and positive values correspond to a joint electron-hole movement, i.e. exciton formation. To rationalize excitonic effects, the exciton wavefunction can be decomposed as

$$
\chi_{e x c}\left(r_{h}, r_{e}\right) \approx \phi_{h e}\left(r_{h e}\right) \phi_{C M}(R),
$$


where $\phi_{h e}\left(r_{h e}\right)$ describes the intrinsic electron-hole structure, which is similar to a hydrogen atom but with different effective masses, and $\phi_{C M}(R)$ describes the center-of-mass $(\mathrm{CM})$ movement of the neutral exciton within the molecular potential. ${ }^{22,23}$ To visualize the decomposed exciton wavefunction in terms of the joint electron-hole distribution in space we employ so-called electron-hole correlation plots (cf. Refs. 7,22,24). The probability of an electron being promoted from one molecular site to another is encoded in grey scale.

All calculations are performed with Q-Chem $4.3 .{ }^{25}$ For the TDDFT calculations, the geometries are optimized in the corresponding ground state for each functional using Alrichs' $\mathrm{SV}(\mathrm{P})$ basis set. ${ }^{26}$ Seven functionals are selected with different amounts of non-local orbital exchange (NLX): (a) $\mathrm{PBE}^{27}$ as representative of local generalized-gradient-approximationtype functionals without non-local orbital exchange; (b) three different global hybrid functionals including: B3LYP (21\%), ${ }^{28,29}$ PBE0 (25\%), ${ }^{30}$ and M06-2X (54\%); ${ }^{31}$ (c) three different long-range corrected (LRC) functionals CAM-B3LYP $(19-65 \%),{ }^{32} \omega \mathrm{PBE}(0-100 \%),{ }^{33}$ and $\omega \mathrm{B} 97(0-100 \%)^{34}$ (fraction of NLX in parenthesis). For the configuration interaction singles (CIS) calculations, the geometries are optimized at the Hartree Fock (HF) level of theory and for the algebraic-diagrammatic construction for the polarization propagator $(\mathrm{ADC}(2))$ calculations, ${ }^{35-37}$ the geometries are optimized with $\omega$ B97. Conjugation lengths are determined by measuring the distance of the outermost atoms for each system for the B3LYP-optimized geometry and are presented in the supporting information. This quantity is not a unique measure for the extent of the $\pi$-system, nevertheless it is accurate enough for a rough estimate for the HOMO and LUMO sizes, since they are in all cases completely delocalized over the entire molecules. Electron-hole correlation plots are created using TheoDORE 1.1.4 ${ }^{38}$ with fragments representing individual phenyl rings as defined in Fig. 1 in the supporting information.

In the following, the first excited state of a set of prototypical, large $\pi$-conjugated molecules is investigated employing exciton analysis. These $\pi$-systems are the fullerene $\mathrm{C}_{60}$, and oligomers of polythiophene $(\mathrm{T})$, poly (para phenylene vinylene) (PPV), ladder-type 
poly(para phenylene) (LPPP) and poly(benzimidazo-benzophenanthroline) (BBL) as shown in Fig. 1.

(a)

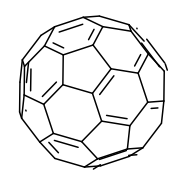

(d) (b)

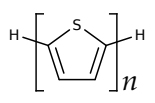

(e)

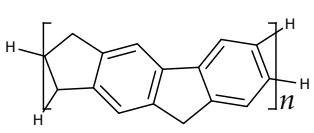

(c)

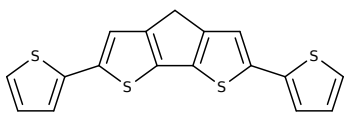

(f)

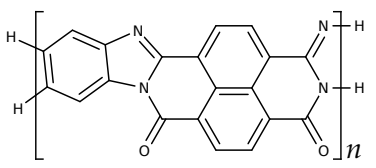

Figure 1: Conjugated systems investigated in this work: (a) $\mathrm{C}_{60}$, (b) polythiophene $(n=$ $4,6,8)$ (c) $4 \mathrm{~TB}$, (d) poly (para phenylene vinylene) $(n=3,5,7)$, (e) ladder-type poly (para phenylene) $(n=1-4,6,8)$, (f) benzimidazo-benzophenanthroline polymer $(n=3-6){ }^{39}$

In Fig. 2 (a) the exciton size for all molecules is plotted against the conjugation length. The first remarkable observation is a uniform exciton size scaling (i.e. the growth of exciton size with the conjugation length, cf. Refs 40,41). This uniform scaling is surprisingly independent from the molecular details of this diverse set of $\pi$-conjugated oligomers. However, large differences are observed between the computational methods. While with PBE ( $0 \%$ NLX) the exciton sizes increase linearly with values of about half of the conjugation lengths, for all other functionals which include non-local orbital exchange, $d_{e x c}$ converges to functional-dependent values for long chains. For B3LYP (21\% NLX), a maximum of approx. $13.0 \AA$ is found, while for PBE0 (25\% NLX) $d_{e x c}$ converges against $11.5 \AA$. With $54 \%$ of nonlocal orbital exchange included, the exciton size with M06-2X is about $7.5 \AA$, and $7.0 \AA$ for the long-range corrected CAM-B3LYP (19-65\% NLX). For the LRC functionals $\omega$ PBE and $\omega \mathrm{B} 97$, which admix up to $100 \%$ non-local orbital exchange at large electron-electron separations, the exciton size converges to $5 \AA$. To put these results into perspective, the exciton sizes are calculated with the ab initio methods CIS and ADC(2). The CIS exciton sizes mark a lower limit below the LRC functionals with a maximum of $5.0 \AA$, while $\operatorname{ADC}(2)$ closely resembles CAM-B3LYP with $7.0 \AA$. For semi-empirical methods, an even lower exciton size of $4 \AA$ is found for ladder-type para phenylene oligomers. ${ }^{15}$ Generally, the results confirm the exciton size trends of Refs 41-44. As opposed to Ref. 40, we do not find an indefinite 
linear increase in $d_{e x c}$, but the exciton size quickly levels off. Seemingly, experimental and our theoretical findings disagree, however, their relation is complex, if not even unclear. For example, it remains ambiguous how to determine exciton sizes from experiment and the approach used in Ref. 40 is still under debate. In addition, effects of dielectric screening are not included in our theoretical study. As a consequence, our results represent polymer chains at the boundaries rather than in the bulk. To address these obvious discrepancies, dielectric screening needs to be taken into account in future theoretical efforts. In conclusion, the asymptotic exciton size limit in TDDFT depends almost exclusively on the amount of non-local orbital exchange included in the functional. This nicely falls into place considering that this value determines the strength of the Coulomb interaction between the electron and hole, cf. Eq. (2).

The results for the hole sizes $\sigma_{h}$ are shown in Fig. 2 (b). The electron sizes behave analogously (see Fig. 2 of SI). In contrast to the exciton sizes $\left(d_{e x c}\right)$, the electron and hole sizes increase linearly for all functionals. This leads us to the question of how to rationalize the discrepancy between $d_{e x c}$ and $\sigma_{h}$ ? For this purpose, it is instructive to examine the linear correlation between electron and hole $\left(R_{e h}\right)$ plotted in Fig. $2(\mathrm{c})$. For the pure GGA functional PBE, the values for the correlation coefficients are negative throughout all systems investigated. This indicates that the electron and hole quasi-particles dynamically avoid each other in space, which is rather typical for charge-transfer states than for bound excitons. ${ }^{4,20}$ For all other functionals that include non-local orbital exchange, the electron-hole correlation coefficients are positive. This corresponds to a correlated motion of electron and hole in space, i.e. exciton formation. For the two hybrid functionals B3LYP and PBE0, the electronhole correlation grows linearly with the system size with maxima for the largest conjugated system of 0.65 (B3LYP) and 0.72 (PBE0). For all LRC functionals and M06-2X, there is a strong increase between 5 to $40 \AA$, after which the value is already close to 1 . The CAM-B3LYP results again closely resemble the $\operatorname{ADC}(2)$ results. These trends illustrate how exciton formation emerges for medium to large sized systems. A key message of the results 

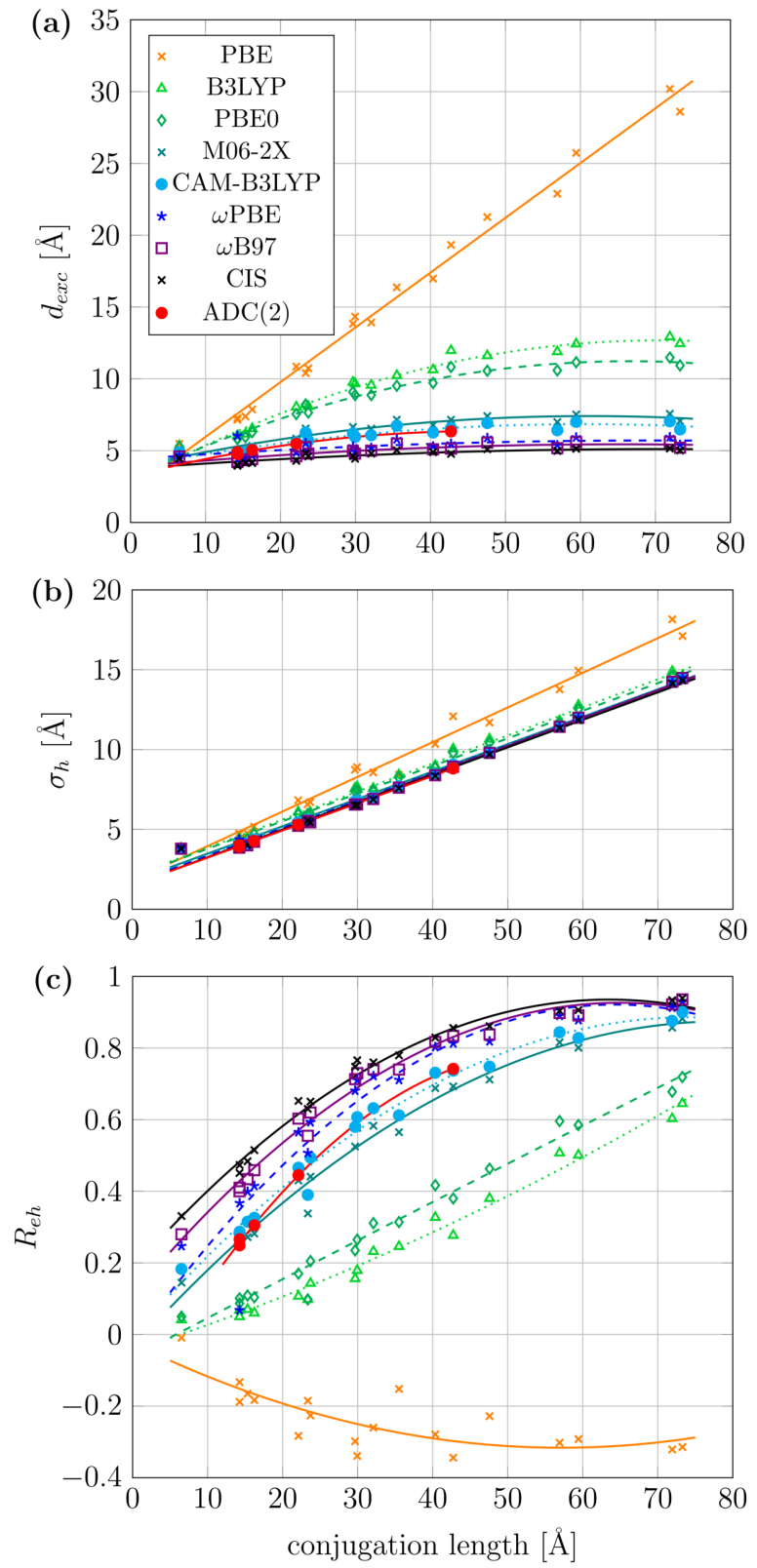

Figure 2: (a) Exciton size $\left(d_{e x c}, \AA\right)$, (b) hole size $\left(\sigma_{h}, \AA\right)$, and (c) correlation coefficient $R_{e h}$ plotted against conjugation length for the first excited singlet state of each system. 
is that the resulting apparent exciton structure can be manipulated from anti-correlated electron-hole pairs to strongly bound excitons just by tuning the $c_{\mathrm{HF}}$ parameter in Eq. (2).

For comparison to the full TDDFT computations reported above, the Tamm-Dancoff Approximation ${ }^{45}$ has been employed to calculate exciton properties for the three functionals PBE, PBE0, and $\omega$ PBE. While the excitation energies of TDDFT and TDA results agree at least up to $0.001 \mathrm{eV}$, the exciton sizes and correlation coefficients stand in reasonable agreement throughout the whole data set, cf. Fig. 3 of SI. In conclusion, the neglect of the de-excitation amplitude $\mathbf{Y}$ and of the $\mathbf{B}$ matrix in Eq. (1) only introduces minor differences in exciton properties.

To further analyze the connection between the correlation coefficient $R_{e h}$, the exciton size $d_{\text {exc }}$ and the hole size $\sigma_{h}$, it is worth to examine their mathematical dependencies. The exciton size can be rewritten as ${ }^{20}$

$$
d_{e x c}=\sqrt{d_{h \rightarrow e}+\sigma_{h}^{2}+\sigma_{e}^{2}-2 \times \operatorname{COV}\left(r_{h}, r_{e}\right)} .
$$

In the case of extended $\pi$-conjugated polymers, we can assume that the vectorial electronhole distance $d_{h \rightarrow e}$ is approx. zero, and that electron and hole sizes are approx. the same $\left(\sigma_{h} \approx \sigma_{e}\right)$. Considering this, Eq. (10) can be simplified to obtain

$$
d_{e x c} \approx \sigma_{h} \sqrt{2\left(1-R_{e h}\right)} .
$$

Eq. (11) allows to predict that for an uncorrelated electon-hole pair the exciton size amounts to $\sqrt{2} \sigma_{h}$, and that it can vary between the values of 0 and $2 \sigma_{h}$ in the cases of positive or negative correlation, respectively. Revisiting Fig. 2, these trends are reproduced as e.g. in the case of PBE, the exciton size is always above $\sqrt{2} \sigma_{h}$, whereas it is always below this value for all other functionals.

To highlight the connection between the values of $R_{e h}$ and the excitonic structure, exciton wavefunctions for the 8LPPP oligomer are visualized in terms of electron-hole correlation 


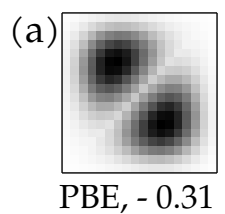

(b)

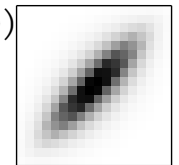

B3LYP, 0.65

(e)

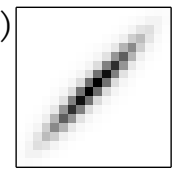

CAM-B3LYP, 0.90 (c)

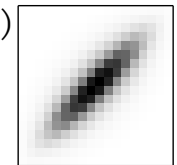

PBE0, 0.72

(f)

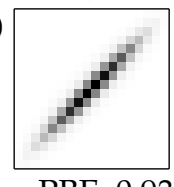

(d)

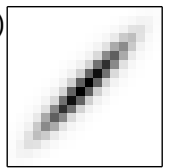

M06-2X, 0.88

(g)

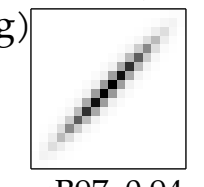

Figure 3: Electron-hole correlation plots and correlation coefficients $R_{e h}$ of the first excited state of 8LPPP calculated using various functionals. The main diagonal going from the lower left to the upper right element represents the probability of an electron being locally excited within one site while promotion between different sites is indicated as off-diagonal elements.

plots $^{7,24,42}$ for all $x c$-functionals, shown in Fig. 3 . For the PBE functional, the elements on the main diagonal (going from lower left to upper right) indicate a probability of almost zero, while the off-diagonal elements show a broad distribution of charge transfer between molecular sites in various distances. This pattern highlights the charge-transfer character of the excited state, ${ }^{8,21,22}$ which coincides with a negative correlation coefficient of -0.31 . On the contrary, for all the other functionals with positive $R_{e h}$ values, the electron-hole correlation plots are dominated by mainly local excitations and charge transfer between neighboring sites. The latter can be attributed to short-range electron-hole repulsion. ${ }^{6}$ These patterns correspond to Wannier-type excitons, for which the intrinsic structure of the electron-hole pair is hydrogenic (for more details see Ref. 22). The off-diagonal width in the electron-hole correlation plots represents the average electron-hole distance (i.e. exciton size), and is controlled by the amount of non-local orbital exchange employed in the respective $x c$-functional in analogy to the values of $d_{e x c}$ and $R_{e h}$.

This decomposition of the exciton wavefunction into an intrinsic part (shown as offdiagonal width of the electron-hole distribution) and an extrinsic part (distribution of the neutral exciton within the molecular potential parallel to main diagonal) allows to rationalize 
the connection between the exciton and MO pictures, cf. Eq. (9). The delocalization of the joint electron-hole pair over the molecular potential generates a non-zero probability for the exciton to be located anywhere in the entire system. This translates into completely delocalized MOs, which themselves, however, are not capable to highlight the underlying exciton structure dominating the excited-state character. At this point it becomes clear why it is misleading to interpret excited states as weakly bound Wannier excitons with large sizes by arguing that the HOMO and LUMO are delocalized over the entire molecule. An exciton can simply not be characterized in the MO picture. To reveal its intrinsic structure, it is imperative to move to a correlated electron-hole representation.

In summary, exciton properties of the energetically lowest singlet excited states of a set of extended $\pi$-conjugated molecules were investigated with TDDFT in combination with a hierarchy of $x c$-functionals as well as the $a b$ initio methods CIS and $\operatorname{ADC}(2)$. With the latter, the exciton size converges against $7 \AA$ in the single chain picture, i.e. in the absence of dielectric screening. In general, the convergence of the exciton size is found to be system-independent, but essentially governed by the amount of non-local orbital exchange in TDDFT. This effect is traced back to the electron-hole correlation which is influenced by the functional-dependent Coulomb attraction between the electron and hole quasiparticles. The problem of pure TDDFT to describe excited states in extended $\pi$-conjugated systems $^{14,46-50}$ hence originates from the inability to describe bound excitonic states. Future work will be dedicated to go beyond the single chain picture, i.e. accounting for inhomogenious environments, geometrical distortions and polaron formation.

\section{Acknowledgement}

S.A.M. acknowledges funding of the Heidelberg Graduate School of Mathematical and Computational Methods for the Sciences (HGS MathComp) and Landesgraduiertenförderung Baden-Württemberg. F.P. acknowledges funding of the VSC Research Center funded by the 
Austrian Federal Ministry of Science, Research, and Economy (bmwfw). Discussions with S. Kraner on the topic of exciton size scaling are gratefully acknowledged.

\section{Supporting Information Available}

Fragmentation scheme of 8LPPP for electron-hole correlation plots in Fig. 3; Conjugation lengths of oligomers; Electron sizes plotted against conjugation lengths; Comparison of exciton sizes and correlation coefficients between TDDFT and TDA. This material is available free of charge via the Internet at http://pubs .acs .org/.

\section{References}

(1) Casida, M. E. In Recent Advances in Density Functional Methods Part I; Chong, D., Ed.; World Scientific: Singapore, 1995; Chapter Time-dependent density functional response theory for molecules, pp 155-192.

(2) Runge, E.; Gross, E. K. U. Density-Funtional Theory for Time-Dependent Systems. Phys. Rev. Lett. 1984, 52, 977-1000.

(3) Dreuw, A.; Head-Gordon, M. Single-Reference Ab Initio Methods for the Calculation of Excited States of Large Molecules. Chem. Rev. 2005, 105, 4009-4037.

(4) Mewes, S. A.; Plasser, F.; Dreuw, A. Exciton Analysis in Time-Dependent Density Functional Theory: How Functionals Shape Excited-State Characters. J. Chem. Phys. 2015, 143, 171101.

(5) Scholes, G. D.; Rumbles, G. Excitons in Nanoscale Systems. Nat. Mater. 2006, 5, 683-696.

(6) Kirova, N. Understanding Excitons in Optically Active Polymers. Polym. Int. 2008, $57,678-688$. 
(7) Plasser, F.; Wormit, M.; Dreuw, A. New Tools For the Systematic Analysis and Visualization of Electronic Excitations. Part I: Formalism. J. Chem. Phys. 2014, 141, 024106 .

(8) Bäppler, S. A.; Plasser, F.; Wormit, M.; Dreuw, A. Exciton Analysis of Many-body Wave Functions: Bridging the Gap Between the Quasiparticle and Molecular Orbital Pictures. Phys. Rev. A 2014, 90, 052521.

(9) Vlček, V.; Eisenberg, H. R.; Steinle-Neumann, G.; Neuhauser, D.; Rabani, E.; Baer, R. Spontaneous Charge Carrier Localization in Extended One-Dimensional Systems. Phys. Rev. Lett. 2016, 116, 186401.

(10) De Roeck, W.; Huveneers, F. Scenario for Delocalization in Translation-Invariant Systems. Phys. Rev. B 2014, 90, 165137.

(11) Hickey, J. M.; Genway, S.; Garrahan, J. P. Signatures of Many-Body Localisation in a System Without Disorder and the Relation to a Glass Transition. arXiv:1405.5780

(12) Yao, N. Y.; Laumann, C. R.; Cirac, J. I.; Lukin, M. D.; Moore, J. E. Quasi Many-body Localization in Translation Invariant Systems. arXiv:1410.7407

(13) Schiulaz, M.; Silva, A.; Müller, M. Dynamics in Many-Body Localized Quantum Systems Without Disorder. Phys. Rev. B 2015, 91, 184202.

(14) Wong, B. M.; Hsieh, T. H. Optoelectronic and Excitonic Properties of Oligoacenes: Substantial Improvements from Range-Separated Time-Dependent Density Functional Theory. J. Chem. Theory Comput. 2010, 6, 37043712.

(15) Rissler, J.; Bässler, H.; Gebhard, F.; Schwerdtfeger, P. Excited States of Ladder-type Poly-p-phenylene Oligomers. Phys. Rev. B 2001, 64, 045122.

(16) Rissler, J. Effective Conjugation Length of $\pi$-Conjugated Systems. Chem. Phys. Lett. 2004, 395, 9296. 
(17) Tretiak, S.; Igumenshchev, K.; Chernyak, V. Exciton Sizes of Conducting Polymers Predicted by Time-Dependent Density Functional Theory. Phys. Rev. B 2005, 71, 033201.

(18) Onida, G.; Reining, L.; Rubio, A. Electronic Excitations: Density-Functional Versus Many-Body Green's-Function Approaches. Rev. Mod. Phys. 2002, 74, 601-659.

(19) Yu, H. S.; Li, S. L.; Truhlar, D. G. Perspective : Kohn-Sham Density Functional Theory Descending a Staircase. J. Chem. Phys. 2016, 145, 130901.

(20) Plasser, F.; Thomitzni, B.; Bäppler, S. A.; Wenzel, J.; Rehn, D. R.; Wormit, M.; Dreuw, A. Statistical Analysis of Electronic Excitation Processes: Location, Compactness, and Electron-Hole Correlation. J. Comp. Chem. 2015, 36, 1609-1620.

(21) Plasser, F.; Lischka, H. Analysis of Excitonic and Charge Transfer Interactions From Quantum Chemical Calculations. J. Chem. Theo. Comp. 2012, 8, 2777-2789.

(22) Mewes, S. A.; Mewes, J.-M.; Dreuw, A.; Plasser, F. Excitons in Poly(para phenylene vinylene): A Quantum-chemical Perspective Based on High-level Ab Initio Calculations. Phys. Chem. Chem. Phys. 2016, 18, 2548-2563.

(23) Barford, W.; Paiboonvorachat, N. Excitons in Conjugated Polymers: Wavefunctions Symmetries, and Quantum Numbers. J. Chem. Phys. 2008, 129, 162716.

(24) Luzanov, A. V.; Zhikol, O. A. Electron Invariants and Excited State Structural Analysis for Electronic Transitions Within CIS, RPA, and TDDFT Models. Int. J. Quant. Chem. 2010, 110, 902-924.

(25) Shao, Y.; Molnar, L. F.; Jung, Y.; Kussmann, J.; Ochsenfeld, C.; Brown, S. T.; Gilbert, A. T.; Slipchenko, L. V.; Levchenko, S. V.; P., O. D. et al. Advances in Quantum Chemical Methods and Algorithms in the Q-Chem 3.0 Program Package. Phys. Chem. Chem. Phys. 2006, 8, 3172. 
(26) Schäfer, A.; Horn, H.; Ahlrichs, R. Fully Optimized Contracted Gaussian Basis Sets For Atoms Lithium to Krypton. J. Chem. Phys. 1992, 97, 2571-2577.

(27) Perdew, J. P.; Burke, K.; Ernzerhof, M. Generalized Gradient Approximation Made Simple. Phys. Rev. Lett. 1996, 77, 3865-3868.

(28) Becke, A. D. DensityFunctional Thermochemistry. III. The Role of Exact Exchange. J. Chem. Phys. 1993, 98, 5648-5652.

(29) Lee, C.; Yang, W.; Parr, R. G. Development of the Colle-Salvetti Correlation-Energy Formula Into a Functional of the Electron Density. Phys. Rev. B 1988, 37, 785-789.

(30) Adamo, C.; Barone, V. Toward Reliable Density Functional Methods Without Adjustable Parameters: The PBE0 Model. J. Chem. Phys. 1999, 110, 6158-6170.

(31) Zhao, Y.; Truhlar, D. G. The M06 Suite of Density Functionals for Main Group Thermochemistry, Thermochemical Kinetics, Noncovalent Interactions, Excited States, and Transition Elements: Two New Functionals and Systematic Testing of Four M06-class Functionals and 12 Other Functionals. Theor. Chem. Acc. 2008, 120, 215-241.

(32) Yanai, T.; Tew, D. P.; Handy, N. C. A New Hybrid Exchange-Correlation Functional Using the Coulomb-Attenuating Method (CAM-B3LYP). Chem. Phys. Lett. 2004, 393, $51-57$.

(33) Henderson, T. M.; Janesko, B. G.; Scuseria, G. E. Generalized Gradient Approximation Model Exchange Holes for Range-Separated Hybrids. J. Chem. Phys. 2008, 128.

(34) Chai, J.-D.; Head-Gordon, M. Systematic Optimization of Long-Range Corrected Hybrid Density Functionals. J. Chem. Phys. 2008, 128, 084106.

(35) Schirmer, J. A New Approximation Scheme for the Polarization Propagator. Phys. Rev. A 1982, 26, 2395-2416. 
(36) Trofimov, A. B.; Schirmer, J. An Efficient Polarization Propagator Approach to Valence Electron Excitation Spectra. J. Phys. B 1995, 28, 2299-2324.

(37) Dreuw, A.; Wormit, M. The Algebraic Diagrammatic Construction Scheme for the Polarization Propagator for the Calculation of Excited States. WIREs Comput. Mol. Sci. 2015, 5, 82-95.

(38) Plasser, F. TheoDORE: A Package for Theoretical Density, Orbital Relaxation, and Exciton Analysis; available from http://theodore-qc.sourceforge.net.

(39) ChemAxon, Structures created with Marvin 6.1.2,(http://www. chemaxon. com). 2013.

(40) Knupfer, M.; Fink, J.; Zojer, E.; Leising, G.; Fichou, D. Universal Exciton Size Scaling in Conjugated Systems. Chem. Phys. Lett. 2000, 318, $585-589$.

(41) Kraner, S.; Scholz, R.; Plasser, F.; Koerner, C.; Leo, K. Exciton Size and Binding Energy Limitations in One-Dimensional Organic Materials. J. Chem. Phys. 2015, 143, 244905.

(42) Tretiak, S.; Mukamel, S. Density Matrix Analysis and Simulation of Electronic Excitations in Conjugated and Aggregated Molecules. Chem. Rev. 2002, 102, 3171-3212.

(43) Igumenshchev, K. I.; Tretiak, S.; Chernyak, V. Y. Excitonic Effects in a Time-dependent Density Functional Theory. J. Chem. Phys. 2007, 127, 114902.

(44) Plasser, F. Entanglement Entropy of Electronic Excitations. J. Chem. Phys. 2016, 144, 194107.

(45) Hirata, S.; Head-Gordon, M. Time-Dependent Density Functional Theory Within the Tamm-Dancoff Approximation. Chem. Phys. Lett. 1999, 314, 291-299.

(46) Richard, R.; Herbert, J. Time-Dependent Density-Functional Description of the 1La State in Polycyclic Aromatic Hydrocarbons: Charge-Transfer Character in Disguise? J. Chem. Theo. Comp. 2011, 7, 1296-1306. 
(47) Cai, Z. L.; Sendt, K.; Reimers, J. R. Failure of Density-Functional Theory and TimeDependent Density-Functional Theory for Large Extended $\pi$ Systems. J. Chem. Phys. 2002, 117, 5543 .

(48) Grimme, S.; Parac, M. Substantial Errors from Time-Dependent Density Functional Theory for the Calculation of Excited States of Large Systems. Chem. Phys. Chem. 2003, 4, 292-295.

(49) Wang, Y.-L.; Wu, G.-S. Improving the TDDFT Calculation of Low-Lying Excited States for Polycyclic Aromatic Hydrocarbones Using the Tamm-Dancoff Approximation. Int. J. Quantum Chem. 2008, 108, 430-439.

(50) Kuritz, N.; Stein, T.; Baer, R.; Kronik, L. Charge-Transfer-Like $\pi \rightarrow \pi^{*}$ Excitations in Time-Dependent Density Functional Theory: A Conundrum and Its Solution. J. Chem. Theo. Comp. 2011, 7, 2408-2415. 